\title{
Perception of partly occluded objects by young chicks
}

\author{
LUCIA REGOLIN \\ University of Padua, Padua, Italy \\ and \\ GIORGIO VALLORTIGARA \\ University of Udine, Udine, Italy
}

\begin{abstract}
Completion of partly occluded objects is a ubiquitous phenomenon in human visual perception. It is unclear, however, whether it occurs at all in other species: Studies on visual discrimination learning have revealed that animals usually attend to parts and features of the discriminative stimuli rather than to global object properties. We provide here the first demonstration of recognition of partly occluded objects in a bird species, the domestic chick Gallus gallus, using the naturalistic setting made available by filial imprinting, a process whereby young birds form attachments to their mothers or some artificial substitute. In Experiment 1, newborn chicks were reared singly with a red cardboard triangle, to which they rapidly imprinted and therefore treated as a social partner. On Day 3 of life, the chicks were presented with pairs of objects composed of either isolated fragments or occluded parts of the imprinting stimulus. Chicks consistently chose to associate with complete or with partly occluded versions of the imprinting object rather than with separate fragments of it. Similarly, in Experiment 2, chicks reared with a partly occluded triangle chose to associate with a complete triangle rather than with a fragmented one, whereas chicks reared with a fragmented triangle chose to associate with a fragmented triangle and not with a complete one. Newborn chicks thus appear to behave as if they could experience amodal completion.
\end{abstract}

We all live in a world of objects, mostly opaque, whose fronts hide their backs and, depending on an observer's vantage point, may partly hide some other objects and, in turn, be partly hidden by others. Nevertheless, as humans, our perception hardly suffers when objects are partly hidden. We do not perceive only the pieces or fragments of objects: the parts that are directly visible usually suffice for recognition of the whole object. Although knowledge and memory of how objects are formed may sometimes play a part in this recognition, it is widely accepted that they are secondary to a more fundamental perceptual process of "amodal" completion (see Kanizsa, 1979; Michotte, 1963; Michotte, Thinés, \& Crabbé, 1964), which generates a genuine phenomenal presence of the nonvisible parts, and which depends on detection of certain configurational relationships in visual scenes, such as the alignment of visible parts and similarities in their colors and textures.

We are grateful to Mario Zanforlin for providing facilities, suggestions, and constant encouragement. We also wish to thank Paola Bressan and two anonymous referees for helpful comments. Part of this work was done by the first author in partial fulfillment of the requirements for the PhD degree in the Psychology Department of the University of Padua. This paper is dedicated to the memory of Gaetano Kanizsa, with whom one of us (G.V.) had the pleasure of discussing the problem of studying amodal completion in animals. Correspondence should be addressed to G. Vallortigara, Università di Udine, Istituto di Filosofia, Pedagogia, Didattica delle Lingue Moderne, Via Antonini 8, 33100 Udine, Italy (c-mail: giorgio.vallortigara@ifp.uniud.it).
There have been very few studies of animal recognition of partly occluded objects, and their results appear contradictory. After training pigeons to respond to a triangle, Cerella (1980) found that responses to an amputated triangle exceeded those to a partially occluded triangle. He also reported that after learning to discriminate figures of Charlie Brown, pigeons responded to pictures representing only parts of Charlie Brown's figure but also emitted many responses to random mixtures of these parts. These results seem to suggest that pigeons perceive complex stimuli as an assembly of local features, and that response to partly occluded objects depends only on visual information remaining after fragmentation of the stimulus.

In contrast, Towe (1954) reported that, after training pigeons to discriminate between a triangle and a square, correct responding was maintained even after the two stimuli were partly concealed using a rectangular bar at several different orientations and positions.

More recently, Kanizsa, Renzi, Conte, Compostela, and Guerani (1993) obtained some evidence for amodal completion in mice. Mice were trained to discriminate between complete and amputated disks. After reaching criterion, the mice performed test trials in which outlined rectangles were either exactly juxtaposed or only placed close to the missing sectors of the disks in order to produce or not produce the impression (to a human observer) of an occlusion of the missing sectors by the rectangles. Mice responded as if they were experiencing amodal completion, but alternative interpretations could not be excluded (e.g., sim- 
ilarity judgments between training and transfer stimuli based on the presence of $\mathrm{T}$ and $\mathrm{L}$ junctions). The authors concluded that their data were at least not incompatible with the hypothesis of amodal completion.

One reason for this unsatisfactory state of affairs may lie in the use of discrimination-learning paradigms. It is known that during discrimination learning animals often tend to pay attention to parts and features of the discriminative stimuli (sometimes because of natural predispositions and preferences) rather than to their more global object properties (see Ingle, 1978, and Vallortigara, Zanforlin, \& Compostella, 1990, for discussions of this issue).

We thus reasoned that filial imprinting, the learning process through which the young of some animals (usually of precocial species) come to recognize an object by being exposed to it, could provide a much more interesting and ecologically valid way in which to study the perception of partly occluded objects.

Soon after hatching, visually naive chicks approach a wide range of conspicuous objects. If chicks are exposed to a particular object for some time, they learn its characteristics. When their preferences are subsequently tested, the chicks selectively approach the "imprinted" object and avoid a novel object (see Bolhuis, 1991, \& Horn, 1985, for reviews).

In a natural environment, the mother hen and the chicks' companions are likely to be frequently partially concealed by grass and vegetation, and it is important for the chick not to lose contact even when only parts of its social fellows are directly visible. Although acoustic stimuli are surely crucial for orientation, there is some evidence, from detour studies, that chicks can respond even in the presence of only visual stimuli associated with a partially concealed imprinted object (see Regolin, Vallortigara, \& Zanforlin, 1994, 1995, in press). Of course, one can argue that chicks are, in these cases, responding to parts and features without recognition of the global object. Yet, we think that this is unlikely. Contrary to the original tenet of Lorenz (1937), according to which imprinting leads to recognition of, and a preference for, the rearing species, it is now clear that imprinting is likely to be concerned with learning the characteristics of individuals (see Bolhuis, 1991). This requires response not only to general and salient preselected features, but also to those specific and idiosyncratic properties and distributions of unique features that characterize a particular individual. Since it is likely to be difficult or impossible to choose, in advance, the features that may be different in any particular stranger, it is necessary to have available descriptions of familiar fellows, which should be as complete as possible and which should be compared as fully as possible with those of the new chick (see Vallortigara \& Andrew, 1994).

Young chicks can recognize familiar and unfamiliar conspecifics even after only a few hours of social rearing (Zajonc, Wilson, \& Rajecki, 1975). They have been proved able to recognize individuals using stuffed hens (Johnson \& Horn, 1987), slides representing conspecifics (Ryan, 1982), and artificial objects (Vallortigara \& Andrew, 1991). In particular, it has been shown that 3-day- old females faced with a choice between a cagemate and a conspecific stranger of the same breed prefer to associate with the cagemate (Vallortigara, 1992a). Furthermore, in choosing among table-tennis balls with bars on one face at various orientations, female chicks always chose the ball with a bar at the same orientation as they had encountered in the rearing condition (see Vallortigara \& Andrew, 1991). In this study, we take advantage of this sophisticated visual responsiveness to investigate recognition of partly occluded imprinted objects.

\section{EXPERIMENT 1}

\section{Methods}

Subjects. The subjects were 152 female' autosexed Hybro (a local commercial variety derived from the White Leghorn breed) chicks obtained from a commercial hatchery when they were only a few hours old. They arrived at the laboratory in closed dark boxes at around $1100-1200 \mathrm{~h}$, and were placed singly into rearing cages ( $22 \mathrm{~cm}$ wide $\times 40 \mathrm{~cm}$ high $\times 30 \mathrm{~cm}$ deep) with the imprinting stimulus. The cages were illuminated by fluorescent lamps, and the animals were maintained at a controlled temperature $\left(30^{\circ}-35^{\circ} \mathrm{C}\right)$ with free access to food and water.

Apparatus and Procedure. The testing apparatus (see Vallortigara \& Andrew, 1991, for a detailed description) consisted of a cage identical in size and color to the rearing cages. During the test (which lasted $6 \mathrm{~min}$ ), each bird chose between two stimuli placed at opposite ends of the cage. Using a computer-driven event recorder, we measured how long the chick spent in each third of the cage (middle and ends) (see Vallortigara \& Andrew, 1991, for details of the procedure). The test was started in the early morning of the chicks' 3rd day of life.

Stimuli. The chicks were reared singly with a freely moving cardboard red equilateral triangle $(5.5 \mathrm{~cm}$ per side) that was suspended by a fine thread at about the chick's head height. Previous work (see Vallortigara, 1992x; Vallortigara \& Andrew, 1991) had shown that young chicks imprint quite well with this sort of stimulus. At test, on Day 3, separate groups of chicks were presented with pairs of stimuli located at the opposite ends of the test cage. The stimuli were made of colored cardboard (the same used for rearing) and were pasted on the opposite (shorter) walls of the cage at the height set during rearing. The positions of the two stimuli and which of the two long walls of the cage the chick faced on entry were balanced across individuals. Four different experimental conditions were devised (see Figure 1). In the first, chicks $(n=45)$ were faced

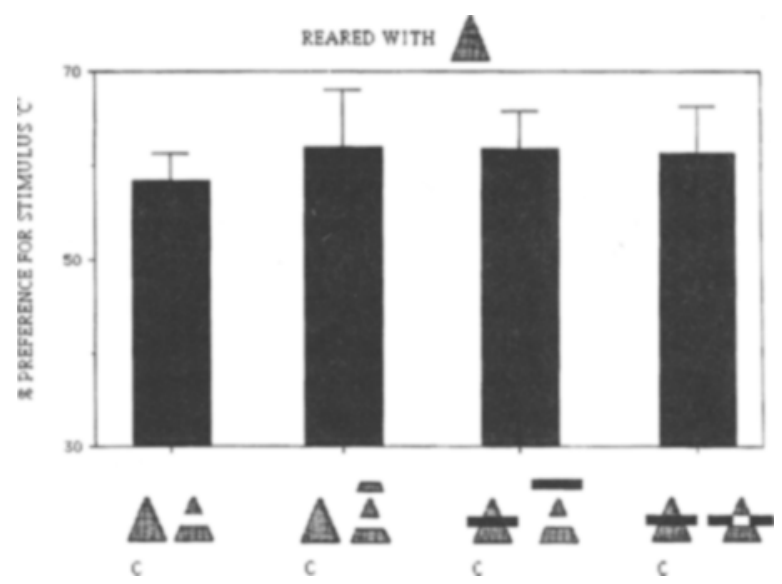

Figure 1. Preference for the complete (or amodally completed) stimulus (C). Group means $( \pm S E$ ) are shown. 
with a triangle identical to the one in the rearing cage and with one in which a central part was missing (the height of the missing part was $1.5 \mathrm{~cm})$. The second condition $(n=35)$ was similar except that the missing central part was displaced above the amputated triangle. The last two conditions were specifically concerned with amodal completion. In the third $(n=44)$, a triangle partly occluded by a black bar ( $10 \mathrm{~cm}$ long and $1.5 \mathrm{~cm}$ high) was contrasted with an amputated triangle with a nonoccluding similar bar (see Figure 1). In the fourth condition $(n=29)$, the bar was split into two identical halves placed in correspondence with the missing part of the triangle (Figure 1).

Data analysis. The stimulus that appears modally or amodally completed according to the human visual experience was arbitrarily chosen as a reference stimulus and labeled "C." Time spent near one or the other of the two stimuli was analyzed as: (times near stimulus $\mathrm{C} /$ total time near the two stimuli) $\times 100$. Thus, significant departures from chance level $(50 \%)$ indicated either choice for stimulus "C" $(>50 \%)$ or choice for stimulus "not C" $(<50 \%)$. Departures from chance levels were estimated by two-tailed one-sample $t$ tests. (See Bateson, 1991, for a discussion of the data-analyzing methods used in recognition tests that make use of filial imprinting.)

\section{Results}

The results are shown in Figure 1. When faced with a choice between the complete and the amputated triangle, chicks clearly preferred to associate with the complete triangle, the stimulus they had been reared with $[t(44)=$ $2.543, p \leq .01]$. The choice did not seem to be due to a generic preference for figures with more extended red areas: when the amputated part of the triangle was dislocated so as to produce a "scrambled" triangle (see Figure 1 ), chicks still preferred the complete triangle $[t(34)=$ $2.115, p<.05]$.

When faced with a partly occluded triangle and an amputated triangle (both stimuli with exactly the same amount of red and black areas), the chicks clearly chose the partly occluded triangle $[t(43)=2.667, p \leq .01]$.

Note that this last condition is, for an organism that could perceive occluded objects as amodally completed, identical to the first condition. The only alternative explanation to amodal completion would be to suppose that chicks have a preference for visually compact objects as opposed to fragmented ones (though there is no biological reason for such a preference). We tried to test this by devising a situation in which the occluding bar was split in half and juxtaposed to the missing part of the triangle (see Figure 1, rightmost column). In this case, the background in the middle region of the triangle was clearly visible (much more clearly so than in the schematic representation of Figure 1 due to the difference in the thickness of the cardboard figure), as was the fragmented nature of the triangle, but there was compactness of the overall figure, particularly with respect to external boundary continuity. Even in this case, however, chicks showed a preference for the occluded triangle $[t(28)=2.306, p<.05]$.

Time spent in the middle of the cage was $38 \%$ on average, mainly due to the few minutes of an immobility reaction (freezing) that occurred when the animals were first placed in the test cage. Times spent in the middle in the four conditions (mean percentages $\pm S E$ ) were: $41 \%$ $\pm 4.3 \%, 33 \% \pm 3.9 \%, 40 \% \pm 4.6 \%$, and $40 \% \pm 6.3 \%$,
respectively.

\section{EXPERIMENT 2}

In Experiment 1, we obtained evidence for the chick's ability to recognize a familiar and highly attractive object when part of the object was concealed. We wondered whether the opposite outcome could also be observed: would chicks exposed to a concealed triangle prefer a complete triangle to a fragmented one? The following experiment was devised to address this issue.

\section{Method}

Subjects. The subjects were 59 female Hybro chicks. Rearing conditions were the same as in Experiment 1.

Apparatus and Procedure. The apparatus and the general procedure were similar to those of Experiment 1. This time, however, the stimulus for imprinting was attached to one of the shorter walls of the rearing cages, at about head height for the chick. (This was necessary because in one of the conditions there were separate parts that could not be freely suspended as could compact stimuli.) The imprinting stimulus (see Figure 2: rearing conditions) consisted either of a red triangle partly covered by a black bar placed horizontally at about midline of the triangle's height $(n=29)$ or of an amputated triangle that consisted of the visible parts of the occluded triangle $(n=30)$. The triangle and the bar were identical to those used in Experiment 1. At test on Day 3, the chicks reared with the occluded triangle and the chicks reared with the amputated triangle were faced with a complete and an amputated triangle (for chicks reared with the occluded triangle, the bars remained present-albeit moved to above the triangles - in both discriminative stimuli in order to minimize change from the rearing conditions, see Figure 2).

\section{Results}

At test, the chicks reared with the occluded triangle preferred the complete over the fragmented triangle $[t(28)=2.967, p<.01$; see Figure 2). This choice cannot be due to a generic preference for the stimulus with a larger colored area (a hypothesis that was also dismissed in the previous experiment), because chicks reared with

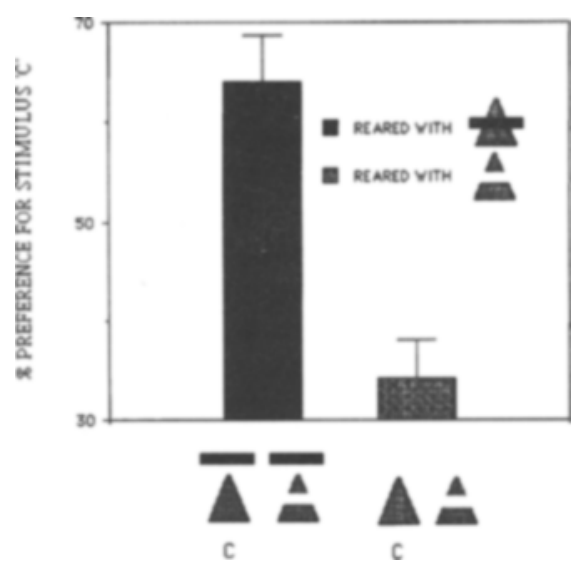

Figure 2. Preference for the complete stimulus (C) as a function of the rearing conditions. Group means $( \pm S E)$ are shown. 
the fragmented triangle did indeed prefer the fragmented (smaller red area) to the complete (larger red area) triangle $[t(29)=-4.033, p<.001$; Figure 2). Similarities between rearing and testing conditions evaluated on the basis of $T$ and $L$ junctions could also be excluded: in chicks reared with the fragmented triangle (L junctions), there were only $\mathrm{L}$ junctions in both discriminative stimuli, and in chicks reared with the occluded triangle ( $\mathrm{L}$ and $\mathrm{T}$ junctions), there were also only $\mathrm{L}$ junctions in both discriminative stimuli.

Times spent in the middle of the cage (mean percentages $\pm S E$ ) were $41 \% \pm 5.9 \%$ (reared with the occluded triangle) and $38 \% \pm 5.6 \%$ (reared with the fragmented triangle).

\section{Discussion}

Even though there could be possible alternative explanations for every single experimental piece of the present work, the overall evidence seems to favor the idea that birds do possess visual abilities very similar to those found in human visual perception of partly occluded objects. This is not surprising. The visual system of vertebrates has probably evolved only once, so that its basic operating principles are likely to be common to all classes. It is now clear that the visual capabilities of birds rival, and in some cases exceed, those of primates (Hodos, 1993; Vaughan \& Greene, 1984), and that the apparently striking differences in the morphology of avian and mammalian visual systems mask an extraordinary degree of similarity (see Karten \& Shimizu, 1989; see also Johnson \& Morton, 1991, for specific parallels between chick and human vision). It would be interesting, however, to consider some reasons that could lie behind previous, unsuccessful attempts to demonstrate recognition of partly occluded objects in birds.

Cerella (1980) provided evidence that pigeons trained to discriminate figures of Charlie Brown learned a set of separate and independent features, associated with reinforcement, of a stimulus that was meaningless to them (see the introduction to the present paper). Yet, we know that a concept (and a visual concept too) is a tool for communication among members of a society (Wittgenstein, 1953); thus, although it is likely that the concept of a conspecific would have a distinct functional value for a pigeon, it is unlikely that the same would be true for the concept of Charlie Brown (see also Watanabe, Lea, \& Dittrich, 1993). And, in fact, when pigeons were trained to discriminate images of conspecifics, they showed a completely different discriminative behavior. Watanabe and Ito (1991) trained pigeons to discriminate color slides of two different conspecific individuals, and then tested them with the full face, separate parts, and randomly connected parts of the positive-stimulus bird. What they found, perhaps unsurprisingly, was that, in this case, pigeons emitted very few responses to abnormal, scrambled figures.

It is likely that the use of naturalistic stimuli per se is not crucial. What is crucial is the biological significance of stimuli. So, in our experiments, while red triangles were in no way similar to natural social fellow chicks, they probably became biologically meaningful through the process of imprinting.

Also note that, although imprinting involves a number of processes, including recognition and selective approach to the familiar object, there is no reason to suppose that the recognition process has a sensitive period as the approach response component: adult fowls recognize conspecifics of their flock (Bradshaw, 1991; Candland, 1969) but do not necessarily follow them (see Horn, 1985). Thus, there is no reason to think that our results should be considered limited in their generality because they are associated to the approach response of filial imprinting.

Does recognition of partly occluded objects result from experience of occluding and disoccluding events or from inborn rules of the sort proposed by Gestalt psychologists? Since some sort of experience with visual objects is essential with both traditional discrimination-learning experiments and recognition tests following exposure learning, it is difficult to definitely answer this question. Nonetheless, it is unclear how chicks in our experiments would have "learned" to recognize partly occluded objects. They remained in complete darkness from hatching to their arrival at the laboratory. Then they remained in a uniformly gray painted cage with the imprinting object for about $48 \mathrm{~h}$. During this time, their experience of occlusive events was very limited. In particular, during rearing before Experiment 1, there was no possibility for the chicks to experience the triangle as partly occluded and/or disoccluded, either by objects or by parts of their bodies.

It has been demonstrated that the chick can perceive subjective contours (Zanforlin, 1981). This finding appears to be crucial in light of the suggestion (see Shipley \& Kellman, 1992) that a single unit-formation process underlies "modal" and "amodal" completion. Similar abilities to perceive subjective contours have been reported for cats (Bravo, Blake, \& Morrison, 1988; DeWeerd, Vandenbussche, De Bruyn, \& Orban, 1990; Redies, Crook, \& Creutzfeldt, 1986) and macaque monkeys (Peterhans \& von der Heydt, 1989; von der Heydt \& Peterhans, 1989; von der Heydt, Peterhans, \& Baumgartner, 1984). It is easy to predict that these species should also manifest recognition of partly occluded objects.

Although the data reported here are consistent with a nativistic concept of the visual perception of partly occluded objects, it is unclear whether they help to disentangle the two different versions of this concept. Gestalt psychologists have stressed that the principles of organization all derive from intrinsic forces, within the nervous system, which are the manifestation of an underlying tendency toward simplicity (Koehler, 1947; Koffka, 1935). An alternative account has been put forward by other authors (e.g., Kellman \& Spelke, 1983), namely, that the ability to recognize occluded objects may emerge in vertebrates without learning, by virtue of an inherent general conception of the physical world (see also Spelke, 1982, 1985). This conception would arise from two separate 
processes in object perception: a "primitive" process, appearing early in development, in which motion alone could indicate unity but not form, and a "rich" process, in which both unity and form are specified (Kellman \& Shipley, 1991). These arguments are based in part on the observation (Kellman \& Spelke, 1983) that 4-month-old human infants can perceive the boundaries of a partly hidden object on the basis of common movements of its end parts (primitive process), but they cannot perceive a connected object when its visible parts are stationary, in spite of color homogeneity, edge alignments, and shape regularity (rich process; see, however, Bower, 1967). Our young chicks did perceive partly occluded objects even when visible parts were kept stationary. However, we are concerned here with a highly precocial species, one that hatches with a large brain and considerable behavioral capacity (Andrew, 1991). Human neonates show no evidence of the perception of partly occluded objects (Slater et al., 1990). Apparently, some development is needed before newborn humans, perhaps the most altricial of species, perceive object unity.

\section{REFERENCES}

ANDREW, R. J. (1991). The chick in experiment: Techniques and tests General. In R. J. Andrew (Ed.), Neural and hehavioural plasticity (pp. 6-11). Oxford: Oxford University Press.

BATESON, P. P. G. (1991). Filial imprinting: Measuring preference and differential responsiveness. In R. J. Andrew (Ed.). Neural and behavioural plasticity (pp. 12-15). Oxford: Oxford University Press.

BolHuis, J. J. (1991). Mechanisms of avian imprinting: A review. Biological Revien's, 66, 303-345.

BowER, T. G. R. (1967). Phenomenal identity and form perception in an infant. Perception \& Psichophrsics, 2, 74-76.

BRADSHAW, R. H. (1991). Discrimination of group members by laying hens Gallus domesticus. Behavioural Processes, 24, 143-151.

Bravo, M., Blake, R., \& Morrison, S. (1988). Cats see subjective contours. Vision Research, 28, 861-865.

Candland, D. K. (1969). Discriminability of facial regions used by the domestic chicken in maintaining the social order. Journal of Comparative \& Phrsiological Psychology, 69, 281-285.

Cerella, J. (1980). The pigeon's analysis of pictures. Pattern Recognition, 12, 1-6.

DeWeerd, P., Vandenbussche, E., De Bruyn, B., \& Orban, G. A. (1990). Ulusory contour orientation discrimination in the cat. Behavioural Brain Research, 39, 1-17.

Hodos, W. (1993). The visual capabilities of birds. In H. P. Zeigler \& H.-J. Bishof (Eds.), Vision, brain, and hehaviour in birds (pp. 63-76). Cambridge, MA: MIT Press.

HoRn, G. (1985). Memory imprinting, and the brain. Oxford: Oxford University Press, Clarendon Press.

INGLE, D. (1978). Mechanisms of shape-recognition among vertebrates. In R. Held, H. W. Leibovitz, \& H. L. Teuber (Eds.), Handbook of sensory physiology VIII (pp. 259-287). Berlin: Springer-Verlag.

JoHnson, M. H., \& HoRN, G. (1987). The role of a restricted region of the chick forebrain in the recognition of individual conspecifics. Behavioural Brain Research, 23, 269-275.

Johnson, M. H., \& MORTON, J. (1991). Biology and cognitive development: The case of face recognition. Oxford. U.K.: Blackwell.

Kanizsa, G. (1979). Organization in vision. New York: Praeger.

Kanizsa, G., Renzi, P., Conte, S., Compostela, C., \& Guerani, L. (1993). Amodal completion in mouse vision. Perception, 22, 713721.

Karten, H. J., \& Shimizu, T. (1989). The origins of neocortex: Connections and laminations as distinct events in evolution. Journal of Cognitive Neuroscience, 1, 291-301.
Kellman, P. J., \& Shipley, T. F. (1991). A theory of visual interpolation in object perception. Cognitive Psychology, 23, 141-221.

Kellman, P. J., \& SPElke, E. S. (1983). Perception of partly occluded objects in infancy. Cognitive Psychology, 15, 483-524.

KoeHLer, W. (1947). Gestalt psychology. New York: Liveright.

KoffKA, K. (1935). Principles of Gestalt psychology. New York: Harcourt, Brace \& World.

LoRENZ, K. (1937). The companion in the bird's world. Auk, 54, 245273

Michotтe, A. (1963). The perception of causality. New York: Basic Books.

Michotte, A., Thinés, G., \& CRABbé, G. (1964). Les complements amodaux des structures perceptives [Amodal complements of perceptual structures]. Louvain, Belgium: Publications Universitaires de Louvain.

Peterhans, E., \& von der Heydt, R. (1989). Mechanisms of contour perception in monkey visual cortex: II. Contours bridging gaps. Journal of Neuroscience, 9, 1749-1763.

Redies, C., Crook, J. M., \& Creutzfeldt, O. D. (1986). Neuronal responses to borders with and without luminance gradients in cat visual cortex and dorsal lateral geniculate nucleus. Experimental Brain Research, 61, 469-481.

Regolin, L., Vallortigara, G., \& Zanforlin, M. (1994). Perceptual and motivational aspects of detour behaviour in young chicks. Animal Behaviour, 47, 123-131.

Regolin, L., Vallortigara, G., \& Zanforlin, M. (1995). Object and spatial representations in detour problems by chicks. Animal Behaviour, 49, 195-199.

Regolin, L., Vallortigara, G., \& Zanforlin, M. (in press). Detour behaviour in the domestic chick: Searching for a disappearing prey or a disappearing social partner. Animal Behaviour.

RYAN, C. M. E. (1982). Concept formation and individual recognition in the domestic chicken (Gallus gallus). Behaviour Analysis Letters, 2, 213-220.

Shipley, T. F., \& Kel.lman, P. J. (1992). Perception of partly occluded objects and illusory figures: Evidence for an identity hypothesis. Journal of Experimental Psychology: Human Perception \& Performance, 18, 106-120.

Slater, A., Morison, V., Somers, M., Mattock, A., Brown, E., \& TAYLOR, D. (1990). Newborn and older infants' perception of partly occluded objects. Infant Behavior \& Development, 13, 33-49.

SPELkE, E. S. (1982). Perceptual knowledge of objects in infancy. In J. Mehler, M. Garrett, \& E. Spelke (Eds.), Perspectives on mental representation (pp. 409-430). Hillsdale, NJ: Erlbaum.

SPELKE, E. S. (1985). Perception of unity, persistence, and identity: Thoughts on infants' conceptions of objects. In J. Mehler \& R. Fox (Eds.), Infant and neonate cognition (pp. 89-113). Hillsdale, NJ: Erlbaum.

TowE, A. L. (1954). A study of figural equivalence in the pigeon. Journal of Comparative \& Physiological Psychology, 47, 283-287.

VALlORTigara, G. (1992a). Affiliation and aggression as related to gender in domestic chicks (Gallus gallus). Journal of Comparative Psychology, 106, 53-57.

VALLORTIGARA, G. (1992b). Right hemisphere advantage for social recognition in the chick. Neuropsychologia, 30, 761-768.

VALLORTIGARA, G., \& ANDREW, R. J. (1991). Lateralization of response by chicks to change in a model partner. Animal Behaviour, 41, 187194.

Vallortigara, G., \& ANDREW, R. J. (1994). Differential involvement of right and left hemisphere in individual recognition in the domestic chick. Behavioural Processes, 33, 41-58.

Vallortigara, G., Zanforlin, M., \& Compostel.la, S. (1990). Perceptual organization in animal learning: Cues or objects? Ethology, 85, 89-102.

Vaughan, W, \& Greene, S. L. (1984). Pigeon visual memory capacity. Journal of Experimental Psychology: Animal Behavior Processes, 10. 256-271

von der Heydt, R., \& Peterhans, E. (1989). Mechanisms of contour perception in monkey visual cortex: I. Lines of pattern discontinuity. Journal of Neuroscience, 9, 1731-1748.

von der Heydt, R., Peterhans, E., \& Baumgartner. G. (1984). I1- 
lusory contours and cortical neuron responses. Science, 224, 12601262.

Watanabe, S., \& Ito, Y. (1991). Discrimination of individuals in pigeons. Bird Behaviour, 9, 20-29.

Watanabe, S., Lea, S. E. G., \& Dittrich, W. H. (1993). What can we learn from experiments on pigeon concept discrimination? In $\mathrm{H}$. P. Zeigler \& H.-J. Bishof (Eds.), Vision, brain, and behavior in birds (pp. 352-376). Cambridge, MA: MIT Press.

Witrgenstein, L. (1953). Philosophical investigations (3rd ed.). Oxford, U.K.: Blackwell.

ZaJonC, R. B., Wilson, W. R., \& RAJECKI, D. W. (1975). Affiliation and social discrimination produced by brief exposure in day-old domestic chicks. Animal Behaviour, 23, 131-138.
ZANFORLIN, M. (1981). Visual perception of complex forms (anomalous surfaces) in chicks. Italian Journal of Psychology, 8, 1-16.

\section{NOTE}

1. We used females because in this strain of chicks females are more inclined to choose the familiar object even when faced with a small degree of change in novel objects; males, in contrast, tend to explore slightly novel objects (see Vallortigara, 1992a; Vallortigara \& Andrew, 1991).

(Manuscript received July 5, 1994;

revision accepted for publication February 14, 1995.) 